\title{
Phototactic Orientation by Amoebae of Dictyostelium discoideum Slug Phototaxis Mutants
}

\author{
By DONAT-P. HÄDER, ${ }^{*}$ KEITH L. WILLIAMS ${ }^{2}$ AND PAUL R. FISHER ${ }^{2}$ \\ ${ }^{1}$ Philipps-Universität, Fachbereich Biologie-Botanik, Lahnberge, D-3550 Marburg, \\ Federal Republic of Germany \\ ${ }^{2}$ Max-Planck-Institut für Biochemie, D-8033 Martinsried, Federal Republic of Germany
}

(Received 28 June 1982 ; revised 16 November 1982)

\begin{abstract}
We examined the phototactic behaviour of individual amoebae of four Dictyostelium discoideum mutants which were altered in their slug phototaxis and thermotaxis. All four mutants were found to be also altered in amoebal phototaxis. This indicates that sensory processing in $D$. discoideum amoebae and slugs is not independent. The mutants were placed in three phenotypic classes. At suboptimal light intensities amoebal phototaxis is bidirectional, deviating right and left from the direction of incident light. Bimodality could be explained by assuming that spatial light gradients produced by internal absorption and scattering during phototaxis depend on the cell geometry in relation to the light direction.
\end{abstract}

\section{INTRODUCTION}

In its multicellular stage (slug) the cellular slime mould Dictyostelium discoideum shows positive phototaxis over a wide range of irradiances (Bonner et al., 1950; Francis, 1964). The mechanism by which the slugs detect the direction of laterally impinging light involves a lens effect (Francis, 1964; Poff \& Loomis, 1973; Häder \& Burkart, 1983). Because the refractive index of the cytoplasm is higher than the surrounding medium, the light rays are focussed on to the distal side of the slug tip. There is reason to believe that the cells on the brighter, distal side produce a higher concentration of a transmitter substance than on the proximal side. Thus, the light gradient is converted into a chemical gradient to which the slug responds with negative chemotaxis (Fisher et al., 1981). The orientation mechanism is further complicated by the observation that slugs do not aim directly toward the light source, but deviate to both sides of this direction (Fisher \& Williams, 1981, 1982).

Dictyostelium amoebae show positive phototaxis (movement toward laterally impinging light) in low irradiances and negative phototaxis (away from light source) in higher irradiances, separated by a small indifference zone (Häder \& Poff, 1979 $a, b$ ). The action spectra for both responses are similar, but differ from the action spectrum for slug phototaxis (Häder \& Poff, $1979 a, b$; Hong et al., 1981). Like slugs, amoebae seem to detect spatial light intensity gradients during phototaxis, but these seem to be established by internal absorption and light scattering rather than by a lens effect as in slugs (Häder \& Burkart, 1983). In spite of these differences in early photosensory events, the signals from amoebal and slug phototaxis may be subsequently processed by a common transduction pathway. To examine whether this is so, we studied the phototaxis behaviour of amoebae of four mutants impaired in slug phototaxis and thermotaxis. The results indicate that signal processing during slug and amoebal phototaxis are not independent.

\section{METHODS}

Amoebae of the parent X22 and the slug phototaxis mutants HU120, HU409, HU410, HU411 (Fisher \& Williams, 1982) were cultivated on sterile $1.5 \%(\mathrm{w} / \mathrm{v})$ water agar plates inoculated with $2 \mathrm{ml}$ samples of an exponential culture of Klebsiella aerogenes growing in a peptone/yeast extract/glucose medium (Sussman, 1966). 
After most of the liquid had evaporated, spores from about 20 sorocarps were transferred on to each fresh plate with a loop. The spores were allowed to germinate and the amoebae to multiply for $48 \mathrm{~h}$ at $21^{\circ} \mathrm{C}$ in darkness. Cells were harvested and evenly distributed on water agar plates $(1 \%)$ at such a concentration $\left(\sim 5 \times 10^{4} \mathrm{~cm}^{-2}\right)$ that individual cells were not disturbed by their neighbours. The amoebae were observed during the first $2 \mathrm{~h}$ after starvation during which no chemotactic effect could be detected. Cell movement on a water agar plate was followed using a Zeiss Universal microscope and a videocamera equipped with an IR sensitive Newvicon tube (National, WV 1350E) (Häder, 1981a,b). The monitoring beam was produced by the built-in light source and a cut-off filter (Schott and Gen.) passing IR wavelengths $>780 \mathrm{~nm}$. The video signal was recorded on a $1 / 2$ inch time-lapse video recorder (National, NV $8030 \mathrm{E}$ ) which allowed for time compression, so that up to $80 \mathrm{~min}$ real time could be played back in 1 min on a video monitor (Sony, PVM-201 CE).

The actinic parallel beam was produced by a $250 \mathrm{~W}$ Leitz halogen slide projector and irradiated laterally at an angle of $15^{\circ}$ above the horizontal plane to minimize mutual shading between the cells as recently described (Häder et al., 1981). The illuminance was controlled by neutral density filters, to avoid changes in the colour temperature (which occur when the lamp voltage is adjusted), and measured by means of a luxmeter (Lange, Berlin) in the plane of the cells. The actinic beam was passed through an IR absorbing filter to avoid the generation of a thermal gradient. An illuminance of $1000 \mathrm{~lx}$ corresponds to an energy fluence rate of about $4.22 \mathrm{Wm}^{-2}$ for this projector as measured with a thermopile (Kipp and Zonen, CAl) connected to a microvoltmeter (Keithley, type 155), previously calibrated against a standard lamp.

During playback, the tracks of the individual cells were traced on a transparent acetate sheet mounted over the video monitor at fixed time intervals. The angular deviation of the paths from the incident light rays was analysed semiautomatically by a goniometer linked to a constant voltage potentiometer and interfaced to a microcomputer (SD Systems). For each experiment (individual strain at a given illuminance) independent tracks of $\sim 800$ amoebae were analysed. The raw data were stored on flexible disk for subsequent mathematical analysis and statistical treatment according to the methods described by Batschelet (1965) using computer programs developed by Häder $(1981 c)$.

\section{RESULTS AND DISCUSSION}

The angular distribution of each population was plotted in circular histograms showing the percentage of cells which moved within a given sector (e.g. between $0^{\circ}$ and $30^{\circ}$, between $30^{\circ}$ and $60^{\circ}$ etc.). From these histograms, we calculated the percentage of organisms moving within $\pm 30^{\circ}$ toward the light source $\left(0^{\circ}\right.$, positive phototaxis) and those moving $\pm 30^{\circ}$ away from the light source $\left(180^{\circ}\right.$, negative phototaxis). The difference between the two subpopulations was plotted for each strain and each illuminance (Fig. 1, hatched bars). Positive values indicate positive phototaxis (more organisms move toward the light than away from it, within $\pm 30^{\circ}$ ) and negative values indicate negative phototaxis. The value on the ordinate can be taken as a rough indicator of the strength of phototactic orientation. The same calculation was repeated for the $\pm 60^{\circ}$ sectors (including the $\pm 30^{\circ}$, solid bar) and the $\pm 90^{\circ}$ sectors (positive half minus negative half, open bars). The results are plotted for the parental strain X22 (Fig. 1a) and each of the mutants (Fig. 1b, $c, d, e$ ) for each light intensity.

$\mathrm{X} 22$ amoebae showed positive phototaxis over a range of light intensities from 1 to $30 \mathrm{~lx}$ with an optimum around $7.5 \mathrm{~lx}$ (Fig. $1 \mathrm{a}$ ). Between 30 and $50 \mathrm{~lx}$ there was a transition to negative phototaxis, and between 100 and $500 \mathrm{~lx}$ a second transition - from negative to positive phototaxis - was observed. This is the first report of this second transition in D. discoideum amoebal phototaxis.

The four mutants studied here show altered phototactic behaviour compared to parent X22 (Fig. $1 a-e$ ) and fall into three phenotype classes. In the first class, represented by HU409, the photoresponse curve was shifted towards lower light intensities (Fig. $1 b$ versus Fig. $1 a$ ). In the second class, comprising HU120 and HU410, the transitions between negative and positive phototaxis seem to be shifted away from the optimum light intensity for negative phototaxis (Fig. $1 c, 1 d$ versus Fig. $1 a$ ). Amoebae of these two mutant strains thus made a transition from positive to negative responses at lower intensity and did not show the second transition negative to positive - even at $1000 \mathrm{~lx}$ (Fig. $1 c, d$ ). The mutant HU411 falls into a third phenotypic class where the pattern of responses is either reversed or dramatically shifted. In contrast to the parent strain X22, HU411 showed transitions from negative to positive, to negative, phototaxis as the light intensity was increased (Fig. $1 e$ versus Fig. $1 a$ ). 


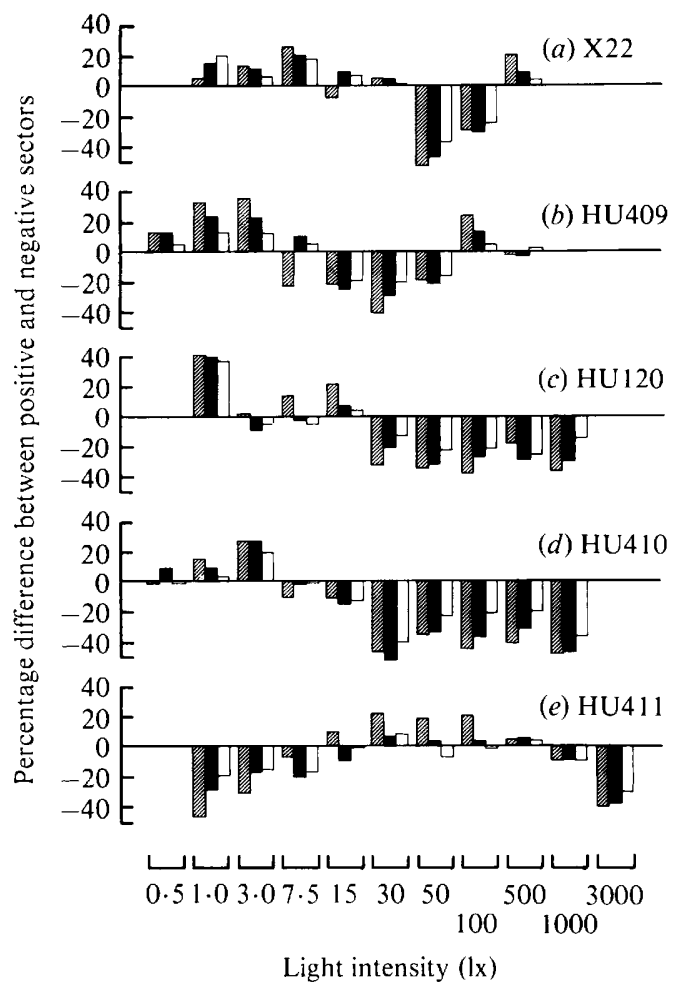

Fig. 1. Orientation of Dictyostelium amoebae of (a) the parental strain X22 and the mutants (b) HU409, (c) HU120, (d) HU410 and (e) HU411 in lateral light. The differences between fractions of cells moving towards the light source with a deviation of $\pm 30^{\circ}$ (hatched bars) $\pm 60^{\circ}$ (solid bars), and $\pm 90^{\circ}$ (open bars) minus those moving away from the light source were calculated as percentage of the sums of the appropriate sectors.

Because the action spectra for positive and negative amoebal phototaxis are the same (Hong et al., 1981), transitions between the two must depend not on integration of signals from different photoreceptors but on how the input signal is subsequently processed. The three classes of mutant behaviour observed here presumably result from mutations that affect the processing logic at different sites in the transduction pathway(s).

Slug phototaxis, like amoebal phototaxis, involves transitions from negative to positive responses (Fisher \& Williams, 1981, 1982; Häder \& Poff, 1979 $a, b$ ). Slugs transit from a negative to a positive response as the angle of deviation from the direction of the light source increases. As a result, slug phototaxis is bidirectional and the preferred direction $( \pm \alpha)$ are the angle at which the transition occurs (Fisher \& Williams, 1981). The mutants HU409, HU120 and HU410 are altered in their transition angle in slug phototaxis (Fisher \& Williams, 1982), in the light intensities at which transitions occur in amoebal phototaxis (Fig. 1) and in the temperature of transition from negative to positive slug thermotaxis (Fisher \& Williams, 1982). This suggests that the transitions in amoebal and slug phototaxis and slug thermotaxis are under common control, although genetic studies are required to confirm that the observed phenotypes in each mutant all result from a single mutation.

Because slug phototaxis is bimodal, we examined whether amoebal phototaxis was also bimodal. For this purpose we recalculated better resolved histograms using $10^{\circ}$ sectors and added the corresponding bilaterally symmetrical sectors (e.g. $0^{\circ}$ to $10^{\circ}$ plus $350^{\circ}$ to $360^{\circ}, 10^{\circ}$ to $20^{\circ}$ plus $340^{\circ}$ to $350^{\circ}$ etc.) and plotted them linearly (Fig. 2). Near the optimal light intensity for positive or negative phototaxis, respectively, orientation was unimodal towards or away from 



Fig. 2. Examples of unimodal phototaxis (a) towards the light source, preferred direction $0^{\circ}(696 \mathrm{HU} 120$ amoebae, $7.5 \mathrm{~lx}$ white light), unimodal phototaxis $(b)$ away from the light source, preferred direction $180^{\circ}(809$ HU120 amoebae, $50 \mathrm{~lx})$ and $(c)$ bimodal phototaxis, preferred directions $145^{\circ}$ and $215^{\circ}(1169$ HU409 amoebae, $50 \mathrm{~lx}$ ). The $10^{\circ}$ sectors left and right from the light direction were summed and plotted as a percentage of the expected value in an ideal random distribution ( $5.556 \%$ of the total population).

the light source (e.g. Fig. $2 a, b)$. At suboptimal light intensities (higher or lower), amoebal phototaxis is bimodal (preferred directions $\pm \alpha$ ), but preferentially positive $\left(\alpha< \pm 90^{\circ}\right)$ or negative $(\alpha$ $> \pm 90^{\circ}$ ). An example is shown in Fig. 2(c).

The histograms furthermore reveal that the accuracy of amoebal phototaxis is very low, producing a 'background noise' of about $2 \%$ in each $10^{\circ}$ sector. Compared to the random distribution both positive and negative phototaxis are very distinct and even in the bimodal distribution (which has a broader maximum) almost twice as many cells move in the $145^{\circ}$ and $215^{\circ}$ sectors as in all other directions. In a totally random distribution one would expèct about $5.6 \%$ of all cells to move within each $10^{\circ}$ sector (times two for right and left half). A statistical analysis of the data (chi-square test, Raleigh test) shows that the data are significant within a probability of $P<0.001$. Though there is a considerable statistical 'noise background', the response is sufficient for an effective long-term orientation of the population. This is most clearly demonstrated by population experiments (Hong et al., 1981) and by light trap techniques (Häder \& Poff, $1979 a, b$ ).

Bidirectional phototaxis by $D$. discoideum amoebae implies that the transitions between negative and positive responses not only depend on light intensity but also on the direction of travel. Migrating amoebae are known to exhibit a temporary morphological polarity (Futrelle $e t$ al., 1982; Gerisch, 1982) - they have a 'head' and a 'tail' - and during phototaxis seem to detect spatial light gradients generated by internal absorption and scattering (Häder et al., 1983). Our results indicate that the degree of absorption and scattering of light depends on the geometry of the cell in relation to the light source direction.

The authors gratefully acknowledge support from the Deutsche Forschungsgemeinschaft to D.-P.H. and thank A. Happel, R. Hill, M. Gläser, I. Hermann, and G. Traxler for skilful technical assistance.

\section{REFERENCES}

BAtSChelet, E. (1965). Statistical methods for the analysis of problems in animal orientation and certain biological rhythms. In Animal Orientation and
Navigation, pp. 61-91. Edited by S. R. Galles, K. Schmidt-Koenig, G. J. Jacob \& R. F. Belleville. Washington: NASA. 
Bonner, J. T., Clark, W. W., JR, NeEley, C. L.. JR \& SLIFKIN, M. K. (1950). The orientation to light and the extremely sensitive orientation to temperature gradients in the slime mold Dictyostelium discoideum. Journal of Cellular and Comparative Physiology 36, 149-158.

FisheR, P. R. \& Williams, K. L. (1981). Bidirectional phototaxis by Dictyostelium discoideum slugs. FEMS Microbiological Letters 12, 87-89.

Fisher, P. R.\& Williams, K. L. (1982). Thermotactic behaviour of Dictyostelium discoideum slug phototaxis mutants. Journal of General Microbiology 128, 965-971.

FisheR, P. R., SMith, E. \& Williams, K. L. (1981). An extracellular chemical signal controlling phototactic behaviour by $D$. discoideum slugs. Cell 23, 799-807.

Francis, D. W. (1964). Some studies on phototaxis of Dictyostelium. Journal of Cellular and Comparative Physiology 64, 131-138.

Futrelle, R. P., Traut, J. \& Mckee, W. G. (1982). Cell behaviour in Dictyostelium discoideum: preaggregation response to localized cyclic AMP pulses. Journal of Cell Biology 92, 807-821.

Gerisch, G. (1982). Chemotaxis in Dictyostelium. Annual Review of Physiology 44, 535-552.

HÄDER, D.-P. (1981a). Effects of inhibitors on photomovement in desmids. Archives of Microbiology 129, 168-172.

HÄDER, D.-P. (1981 b). Electrical and proton gradients in the sensory transduction of photophobic responses in the blue-green alga, Phormidium uncinatum. Archives of Microbiology 130, 83-86.
HÄDER, D.-P. (1981 c). Computer-based evaluation of phototactic orientation in microorganisms. EDP in Medicine and Biology 12, 27-30.

HÄDER, D.-P. \& BURKaRT, U. (1983). Optical properties of Dictoystelium discoideum pseudoplasmodia responsible for phototactic orientation. Experimental Mycology (in the Press).

HÄDER, D.-P. \& POFF, K. L. (1979a). Light-induced accumulations of Dicytostelium discoideum amoebae. Photochemistry and Photobiology 29, 1157-1162.

HÄDER, D.-P. \& PoFF, K. L. (1979b). Photodispersal from light traps by amoebas of Dictyostelium discoideum. Experimental Mycology 3, 121-131.

Häder, D.-P., Colombetti, G., Lenci, F. \& Quaglia, M. (1981). Phototaxis in the flagellates Euglena gracilis and Ochromonas danica. Archives of Microbiology 130, 78-82.

Häder, D.-P., Gerisch, G. \& Claviez, M. (1983). Orientation of Dictyostelium discoideum amoebae to light. Cell Biology International Reports (in the Press).

Hong, C. B., HÄdER, M. A., HÄDER, D.-P. \& POFF, K. L. (1981). Phototaxis in Dictyostelium discoideum amoebae. Photochemistry and Photobiology 33, 373377.

Poff, K. L. \& Loomis, W. F., JR (1973). Control of phototactic migration in Dictyostelium discoideum. Experimental Cell Research 82, 236-240.

Sussman, M. (1966). Biochemical and genetic methods in the study of cellular slime mold development. Methods in Cell Physiology 2, 397-410. 